\title{
Is primary care a neglected piece of the jigsaw in ensuring optimal stroke care? Results of a national study David L Whitford ${ }^{* \dagger 1}$, Anne Hickey ${ }^{\dagger 2}$, Frances Horgan ${ }^{\dagger 3}$, Bernadette O'Sullivan ${ }^{\dagger 2}$, Hannah $\mathrm{McGee}^{\dagger 2}$ and Desmond O'Neill ${ }^{\dagger 4}$
}

Address: ${ }^{1}$ Department of Family \& Community Medicine, Royal College of Surgeons in Ireland-Medical University of Bahrain, PO Box 15503, Adliya, Kingdom of Bahrain, ${ }^{2}$ Department of Psychology, Royal College of Surgeons in Ireland, Dublin 2, Ireland, ${ }^{3}$ School of Physiotherapy, Royal College of Surgeons in Ireland, Dublin 2, Ireland and ${ }^{4}$ Department of Medical Gerontology, Trinity College Dublin, Dublin, Ireland

Email: David L Whitford* - dwhitford@rcsi-mub.com; Anne Hickey - ahickey@rcsi.ie; Frances Horgan - fhorgan@rcsi.ie; Bernadette O'Sullivan - bosullivan9@yahoo.co.uk; Hannah McGee - hmcgee@rcsi.ie; Desmond O'Neill - doneill@tcd.ie

* Corresponding author †Equal contributors

Published: 29 April 2009

BMC Family Practice 2009, 10:27 doi:10.1 I86/147/-2296-10-27
Received: 19 August 2008

Accepted: 29 April 2009

This article is available from: http://www.biomedcentral.com/147I-2296/10/27

(c) 2009 Whitford et al; licensee BioMed Central Ltd.

This is an Open Access article distributed under the terms of the Creative Commons Attribution License (http://creativecommons.org/licenses/by/2.0), which permits unrestricted use, distribution, and reproduction in any medium, provided the original work is properly cited.

\begin{abstract}
Background: Stroke is a major cause of mortality and morbidity with potential for improved care and prevention through general practice. A national survey was undertaken to determine current resources and needs for optimal stroke prevention and care.
\end{abstract}

Methods: Postal survey of random sample of general practitioners undertaken ( $N=204 ; 46 \%$ response). Topics included practice organisation, primary prevention, acute management, secondary prevention, long-term care and rehabilitation.

Results: Service organisation for both primary and secondary prevention was poor. Home management of acute stroke patients was used at some stage by $50 \%$ of responders, accounting for $7.3 \%$ of all stroke patients. Being in a structured cardiovascular management scheme, a training practice, a larger practice, or a practice employing a practice nurse were associated with structures and processes likely to support stroke prevention and care.

Conclusion: General practices were not fulfilling their potential to provide stroke prevention and long-term management. Systems of structured stroke management in general practice are essential to comprehensive national programmes of stroke care.

\section{Background}

Stroke is a major cause of mortality[1] and morbidity[2], and might be argued to be a chronic disease with acute events[3]. Population-based data suggest that acute cerebrovascular events are at least as common as coronary events[4]. The lifetime risk of first-ever stroke from age 55 years in the Framingham cohort was as high as 1 in 5[5]. There is considerable opportunity for primary prevention of stroke: treatments for hypertension[6,7] and non-val- vular atrial fibrillation $[8,9]$ are effective in reducing the risk of stroke, but may not be fully implemented[10]. Observational studies have shown that lifestyle factors such as diet, smoking, exercise, and alcohol intake can predict the risk of stroke [11-13], thereby supporting the adoption of lifestyle risk factor modification. The actuarial risk of recurrent stroke after a first stroke is about 30\% over five years[14], and there is strong evidence of the benefits of anti-platelet therapy[15], blood pressure low- 
ering[16] and lipid lowering[17] in secondary prevention. Despite improvements in the use of secondary prevention medication, there is further scope for achieving more from these medications[18].

General practitioners (GPs) are well placed to implement secondary prevention programmes for stroke. For instance, in an Irish study of 195 discharged stroke patients, the majority ( $87 \%$ ) had seen their GP since hospital discharge, whereas just less than half $(48 \%)$ had been reviewed in hospital outpatient departments[19]. With regard to emergency care, current guidelines recommend all patients with suspected acute stroke are immediately transferred by ambulance to a receiving hospital providing acute stroke services and organised stroke care and that all patients presenting with a recent transient ischaemic attack (TIA) or minor stroke are immediately referred for appropriate urgent specialist assessment and investigation[20]. GPs can play a key role in initiating and directing this rapid response. Finally, GPs can support community-based patient education and primary prevention of stroke since most stroke patients will be community dwellers (for instance, 90\% of Irish stroke patients were community-dwelling before being admitted to hospital with a stroke[21]).

In order for GPs to coordinate optimal stroke prevention and care, a structured approach to the detection and management of chronic disease and risk factors is needed $[22,23]$. Structures to facilitate this include disease registers; clinics for implementing and monitoring the effectiveness of therapy; the use of clinical guidelines or practice protocols to support clinical decisions; and clinical audit to evaluate the effectiveness of treatment provided and stimulate improvement. Information on the capacity of general practice to deliver optimal stroke care is needed. As part of a national evaluation of stroke services in Ireland[24] which assessed community, hospital and nursing home services for stroke, a survey of general practices was undertaken to determine the structures currently in place likely to support stroke prevention and care.

\section{Methods}

\section{Study design, sampling and participants}

We conducted a postal survey among a random sample of GPs taken from a total population of 2,300 GPs in the Republic of Ireland profiled in the Irish Medical Directory 2006-2007 edition. Sampling was conducted using the random selection function in Microsoft Excel. A sample size of 242 GPs provides a $90 \%$ probability that prevalence will be within $5 \%$ of the true value. Based on an expected 50\% response rate, 484 participants were selected.
Selected GPs were sent an invitation letter with an explanation of the value of the survey from the study sponsor the Irish Heart Foundation, a letter of introduction from the research team, the survey instrument, and a stamped addressed envelope. A telephone reminder followed after two weeks if GPs had not returned the questionnaire. GPs were reminded to complete and return the questionnaire, or were given the option to complete the questionnaire by telephone at a time convenient to them. A final reminder questionnaire was sent to non-responders two weeks following the telephone approach (Figure 1). Research ethics approval was granted by the Royal College of Surgeons in Ireland Research Ethics Committee (REC2006:186)

\section{Questionnaire development}

The survey instrument was developed by the research team based on a recent UK general practice survey (Thomas, Chappel, Thomson, \& Rogers, personal correspondence, [see Additional file 1]). The content validity and conceptual clarity of the questionnaire was established for the Irish setting through panel discussion and piloting with ten GPs practicing in five general practices in Dublin. The questionnaire included questions on practice organisation, primary prevention of stroke, acute management of stroke, secondary prevention of stroke, long-term care and rehabilitation.

\section{Analysis}

Data analysis was with SPSS version 15.0 using parametric and non-parametric tests of association. $\mathrm{P}<0.05$ was taken as significant. Variables that were hypothesised to have an impact on delivery of stroke care were membership of Heartwatch (a programme of structured secondary prevention in cardiovascular disease run by $30 \%$ of general practices in Ireland), availability of a practice nurse, and being a training practice. Variables respectively associated with each of these variables at the $5 \%$ level were entered into a logistic regression analysis.

\section{Results}

Of the 448 GPs who were eligible to participate in the survey, 204 did so ( $46 \%$ response rate). There was no difference between responders and non-responders in terms of gender or practice location (i.e. within Dublin or outside Dublin). However, responders were more likely to have qualified after 1980 than non-responders $\left(\mathrm{X}^{2}=8.36,1 \mathrm{df}\right.$, $p=0.004)$. The median age of responders was 49 years (inter-quartile range 42-55). A quarter (24\%) of the responders was single-handed GPs with the median number of doctors per practice being two (range 1-12). The median practice size was 3,000 patients (range 100$25,000)$ and the mean was 4,513 (95\% CI 3,885-5,141) patients. A quarter of the responders $(25 \%)$ were in training practices. Response rates to individual questions 


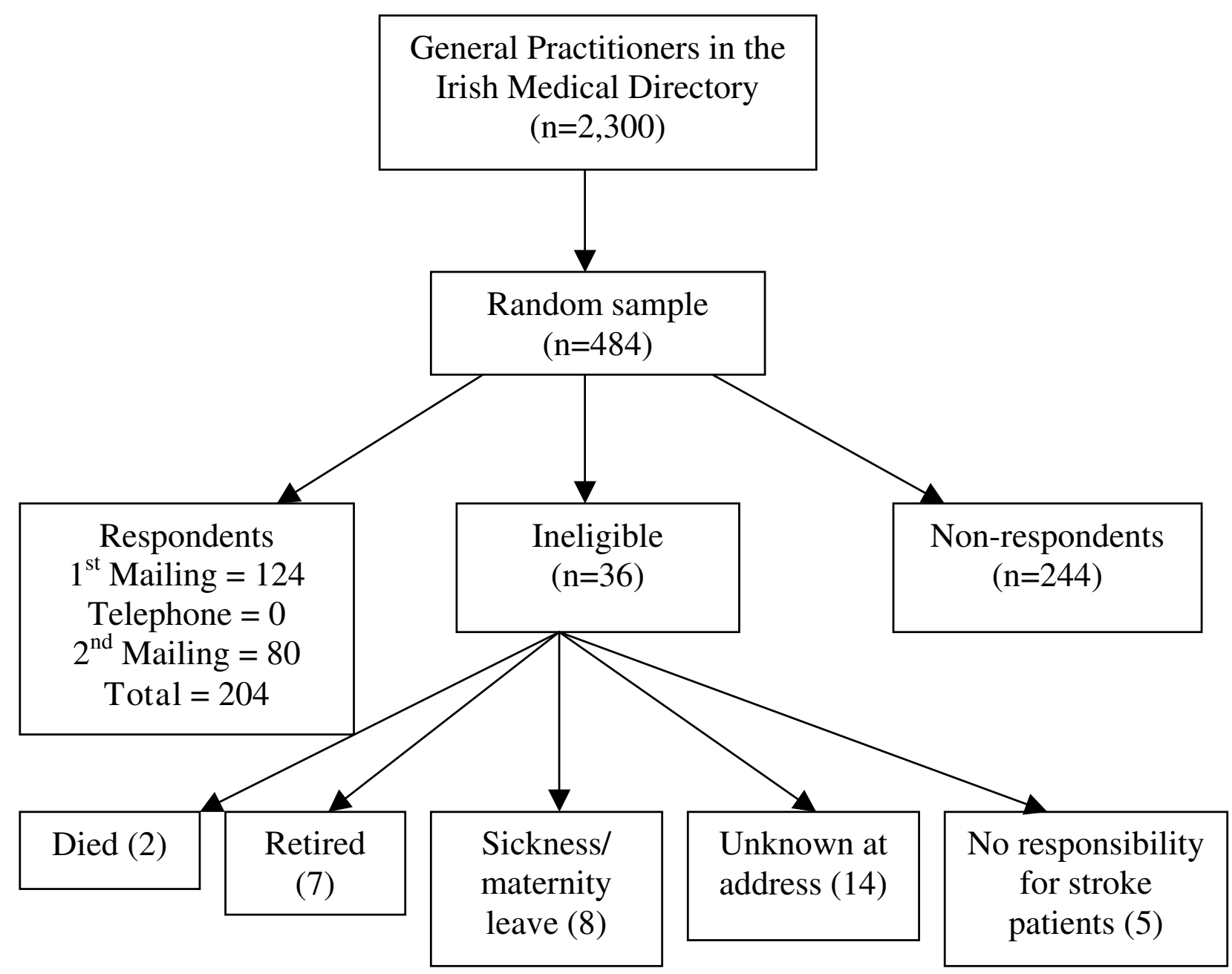

Figure I

ranged from $53 \%$ to $100 \%$ (median $96 \%$ ) with a mean of 94\% (95\%CI 92.6-95.2).

\section{Access to community services}

The majority of responders reported good or excellent access to practice nurses $(75 \%)$, community nurses $(71 \%)$, and physiotherapists (56\%). However, this did not apply to access to other allied health professionals including dieticians $(32 \%)$, social workers $(24 \%)$, psychologists $(15 \%)$, speech and language therapists $(13 \%)$ and occupational therapists (13\%).

\section{Practice organisation}

Practices that were members of the Heartwatch scheme, were training practices and who had practice nurses were more likely to engage in activities likely to prevent strokes (Additional file 2). Variables associated with these three variables respectively were entered into logistic regressions. Membership of the Heartwatch scheme was associated with having registers for atrial fibrillation (Odds ratio (OR) 4.17; 95\% CI 1.58-10.21), diabetes (OR 2.68; 95\% CI 1.17-6.13), and stroke (OR 3.63; 95\% CI 1.19-11.01); and having guidelines for hypertension (OR 2.49; 95\% CI 1.07-5.76) and secondary prevention of stroke (OR 5.61; $95 \%$ CI 1.77-17.74). Being a member of a training practice was associated with having a computerised disease register (OR 4.17; 95\% CI 1.59-10.96), using the computer for audit (OR 4.58; 95\% CI 1.73-12.08), having registers for hypertension (OR 4.02; 95\% CI 1.58-10.21) and atrial fibrillation (OR 4.17; 95\% CI 1.44-12.02) and auditing diabetes care (OR 2.89; 95\% CI 1.13-7.41). Being part of a larger practice ( $>4500$ patients) was associated with having registers for hypertension (OR 1.69; 95\% CI 1.07-2.68) and diabetes (OR 1.59; 95\% CI 1.03-2.46), 
and carrying out a diabetes audit (OR 6.26; 95\% CI 1.1932.88). Having a practice nurse was associated with having a diabetes register (OR 3.29; 95\% CI 1.26-8.58).

\section{Primary prevention of stroke}

Additional file 2 shows aspects of practice structure in relationship to primary prevention of stroke through the management of hypertension, atrial fibrillation and diabetes. Diabetes care was the most organised. The majority of GPs (89\%) reported barriers to the implementation of primary prevention for stroke. These included lack of time $(89 \%)$, inadequate staffing $(86 \%)$, lack of funding $(86 \%)$, lack of screening protocols $(67 \%)$ and lack of risk factor protocols (58\%). The main solutions suggested by GPs to overcome these barriers were the employment of practice nurses and the running of dedicated clinics.

Knowledge amongst GPs about primary prevention of stroke was good with over $90 \%$ being aware that reduction of blood pressure, reduction of cholesterol, use of aspirin with transient ischaemic attacks (TIA) and anticoagulation in patients with AF, as well as carotid endarterectomy in patients with carotid artery stenosis $>70 \%$ were effective in the prevention of stroke. However, almost two thirds (65\%) of GPs incorrectly believed that anticoagulation in patients with a history of TIA was effective in the prevention of stroke.

\section{Secondary prevention of stroke}

The organisation of services for the secondary prevention of stroke was typically poor (Additional file 2). The main barriers listed for secondary prevention were very similar to those recorded for primary prevention, namely lack of time $(60 \%)$, inadequate staffing (57\%), and issues related to funding (33\%). Other barriers listed included lack of protocols/guidelines (17\%) and lack of space (10\%). 87\% of GPs believed that the availability of existing rehabilitation services was inadequate for their stroke patient population.

\section{Management of stroke}

Home management of acute stroke patients was used at some stage by $50 \%$ of responders, accounting for $7.3 \%$ (95\% CI 5.5-9.1) of all stroke patients. However, there was considerable variation among those GPs using home management. The median proportion of patients looked after at home was $10 \%$ but this ranged from one to $80 \%$ (inter-quartile range 5-20\%). Factors influencing the decision for home management included the severity of stroke, the amount of time elapsed since the stroke, the age of the patient, family support, a previous history of stroke and co-morbid disease. Younger GPs $\left(\mathrm{X}^{2}=12.1,3\right.$ $\mathrm{df}, \mathrm{p}=0.007)$, those from larger practices $\left(\mathrm{X}^{2}=6.0,1 \mathrm{df}\right.$, $\mathrm{p}=0.014)$ and those with a lead person for stroke rehabilitation $\left(\mathrm{X}^{2}=6.9,1 \mathrm{df}, \mathrm{p}=0.008\right)$ were less likely to man- age patients at home. Almost all GPs (94\%) managing acute stroke patients at home used aspirin, but $60 \%$ stated they aimed for acute blood pressure reduction after an acute stroke.

Over $85 \%$ of GPs reported that there was no routine liaison from hospitals during admission or leading up to discharge. Similarly, $79 \%$ reported no liaison following discharge from hospital. However, 98\% reported receiving information on discharge medication. Little information was shared with GPs on rehabilitation or community services that had been organised.

\section{Discussion}

This study reveals that there is currently little organisation for the prevention and management of stroke within primary care in Ireland. This is in spite of the clear evidence that up to $60 \%$ of stroke mortality is related to a few preventable vascular risk factors[25] and that structured systems of care are delivering improvements in both these risk factors and in the incidence of stroke[26]. Primary care has an enormous potential to deliver primary prevention of stroke but is failing to rise to this challenge in Ireland. However, there are encouraging signs that practices with a higher level of organisation, such as training practices, practices involved in Heartwatch and practices with good access to practice nurses, were more likely to engage in activities that adequately manage stroke patients.

There is not much evidence from this study of the systematic implementation of healthy lifestyle advice and management of specific risk factors needed to reduce the risk of an initial stroke and the risk of a subsequent stroke. Given the high probability of repeat stroke within five years, this lack of secondary prevention and follow-up in general practice is a cause for concern. The study also highlights the need for improvements in the initial management of acute stroke by some GPs.

\section{Comparison with previous literature}

The acute management of stroke by GPs in Ireland compares favorably with that in the UK where just over half of GPs said they would refer someone with a suspected stroke immediately[27]. However, in the UK almost all general practices have a stroke/TIA register and engage in annual audit that has revealed a steady improvement in most preventive measures[28]. The implementation of structures to support the prevention of cardiovascular disease in Ireland through the Heartwatch scheme has shown similar improvements in preventive measures[29]. A previous study has found the quality of stroke care to be significantly related to aspects of practice organisation including delegation to support staff and compliance with hypertension guidelines[23]. These aspects of practice organisation are absent in many general practices in this 
survey, leaving room for concern at the quality of care received by those surviving stroke.

\section{Strengths and weaknesses}

The response rate in this study was poor but similar to the response rate to many surveys of GPs in Ireland. The smaller sample size provided power of $83 \%$. While the figures in relation to the response rate and power are less than optimal, the study does provide the first national profile from over 200 GPs of stroke management in general practice in Ireland. More recently qualified GPs were over-represented in responders compared to nonresponders. As these GPs may have more up-to-date knowledge in relation to chronic disease management, we caution that the results of this survey may reflect a more positive picture of stroke awareness and care in general practice than exists in reality.

\section{Implications for research and development}

Stroke is often the focus of GP consultations, calculated in one study as over 2 per 1000 encounters[30]. However, it is not yet clear how these consultations could be more effectively utilised in the prevention and management of stroke. Equally, the interface between primary and secondary care poses many challenges in stroke care, but studies of hospital-based stroke coordinators working with primary care physicians have not yet shown evidence of efficacy[31]. The basic requirements of chronic disease management are well established and include the three 'R's: register, recall, and review. However, many challenges beyond these basic requirements remain[32]. For instance, this study found that teamwork among health professionals providing primary care was suboptimal, with access to many stroke team members poor or absent, and it is recommended that any structured scheme of stroke care incorporates an interdisciplinary team-based approach. There is a clear need to establish a system of structured stroke care in general practice that will facilitate the implementation of both primary and secondary prevention of stroke. A model for such a scheme already exists in Ireland in the Heartwatch programme[29] and the Quality Outcomes Framework in the United Kingdom provides an alternative approach[28].

\section{Conclusion}

In summary, general practice is well placed to provide primary and secondary prevention of stroke and long-term management of stroke patients. It is not fulfilling this potential in the Irish health system at present. The involvement of primary care in providing a structured approach to stroke management would appear to be essential to any comprehensive national programme of stroke care. It is proposed that an adequately funded and resourced system of structured stroke care is needed in order to address the deficiencies identified in this study.

\section{Competing interests}

The authors declare that they have no competing interests.

\section{Authors' contributions}

All authors participated in the design of the study. $\mathrm{FH}, \mathrm{AH}$, BOS and DW participated in the coordination and statistical analysis and drafted the manuscript. HM and DON helped to draft the manuscript. All authors read and approved the final manuscript.

\section{Additional material}

\author{
Additional file 1 \\ Questionnaire distributed to General Practitioner. \\ Click here for file \\ [http://www.biomedcentral.com/content/supplementary/1471- \\ 2296-10-27-S1.doc]

\section{Additional file 2} \\ Table S1. \\ Click here for file \\ [http://www.biomedcentral.com/content/supplementary/1471- \\ 2296-10-27-S2.doc]
}

\section{Acknowledgements}

This research was funded by the Irish Heart Foundation in association with the Department of Health and Children, Republic of Ireland.

We thank other Irish National Audit of Stroke Care (INASC) Steering Group members: Professor Seamus Cowman (Nursing and Midwifery RCSI), Dr Sean Murphy (Midland Regional Hospital, Mullingar), Dr Emer Shelley (Epidemiology \& Public Health Medicine RCSI), Professor Miriam Wiley (Economic and Social Research Institute, Dublin), Professor Ronan Conroy (Epidemiology \& Public Health Medicine RCSI).

We thank the INASC Project Research Staff at the Division of Population Health Sciences (Psychology), RCSI: Ms Karen Galligan, Ms Helen Corrigan, Ms Maeve Proctor, Ms Oonagh Mullan, Ms Abigail Henderick, Ms Anna-May Fitzgerald, Ms Philippa Coughlan, Ms Claire Donnellan, Dr Maja Barker and Ms Maeve Royston (General Practice). We thank the Irish Heart Foundation National Stroke Review Group for their support and advice throughout the project. We acknowledge the assistance of Professor Richard Thomson, University of Newcastle, UK and thank the general practitioners who participated.

\section{References}

I. Sarti C, Rastenyte D, Cepaitis Z, Tuomilehto J: International trends in mortality from stroke, 1968 to 1994. Stroke 2000, 31:1588-1601.

2. Bonita R, Solomon N, Broad JB: Prevalence of stroke and strokerelated disability. Estimates from the Auckland stroke studies. Stroke 1997, 28:1898-1902.

3. O'Neill D, Horgan F, Hickey A, McGee H: Stroke is a chronic disease with acute events. BMJ 2008, 336:46I.

4. Rothwell PM, Coull AJ, Silver LE, Fairhead JF, Giles MF, Lovelock CE, et al:: Population-based study of event-rate, incidence, case fatality, and mortality for all acute vascular events in all arterial territories (Oxford Vascular Study). Lancet 2005, 366: |773-1783. 
5. Seshadri S, Wolf PA: Lifetime risk of stroke and dementia: current concepts, and estimates from the Framingham Study. Lancet Neurology 2007, 6: I I06-II I 4.

6. Ebrahim S: Clinical Epidemiology of stroke New York: Oxford University Press; 1999.

7. Neal B, MacMahon S, Chapman N, Blood Pressure Lowering Treatment Trialists' Collaboration: Effects of ACE inhibitors, calcium antagonists, and other blood pressure lowering drugs: results of prospectively designed overviews of randomised trials. Lancet 2000, 356:1955-1964.

8. Atrial Fibrillation Investigators: Risk factors for stroke and efficacy of antithrombotic therapy for atrial fibrillation. Arch Intern Med 1994, I54: 1449-1457.

9. Hart RG, Benavente O, McBride R, Pearce LA: Antithrombotic therapy to prevent stroke in patients with atrial fibrillation: a meta-analysis. Ann Intern Med 1999, I 31:492-50I.

10. White S, Feely J, O'Neill D: Community-based study of atrial fibrillation and stroke prevention. Irish Medical Journal 2004, 97:10-12.

II. Ezekowitz JA, Straus SE, Majumdar SR, McAlister FA: Stroke: Strategies for Primary Prevention. American Family Physician 2003, 68:2379-2386

12. Kurth T, Moore SC, Gaziano M, Kase CS, Stampfer MJ, Berger K, et al.: Healthy lifestyle and the Risk of Stroke in Women. Arch Intern Med 2006, 166: I 403-I 409.

13. Sauvaget C, Nagano J, Allen N, Kodama K: Vegetable and Fruit Intake and Stroke Mortality in the Hiroshima/Nagasaki Life Span Study. Stroke 2003, 34:2355-2360.

14. Burn J, Dennis M, Bamford J, Sandercock P, Wade D, Warlow C: Long-term risk of recurrent stroke after a first-ever stroke. The Oxfordshire Community Stroke Project. Stroke 1994, 25:333-337.

15. Gubitz G, Sandercock P, Counsell C: Antiplatelet therapy for acute ischaemic stroke (Cochrane Review). Oxford: Update Software; 2000.

16. PROGRESS Collaborative Group: Randomised trial of a perindopril-based blood-pressure-lowering regimen among 6,105 individuals with previous stroke or transient ischaemic attack. Lancet 200I, 358:1033-104I.

17. Amarenco P, Bogousslavsky J, Callahan A 3, Goldstein LB, Hennerici $M$, Rudolph $A E$, et al.: High-dose atorvastatin after stroke or transient ischemic attack. N Engl J Med 2006, 355:549-559.

18. Ramsay SE, Whincup PH, Wannamethee SG, Papacosta O, Lennon L, Thomas MC, et al.: Missed opportunities for secondary prevention of cerebrovascular disease in elderly British men from 1999 to 2005: a population-based study. Journal Of Public Health 2007, 29:25I-257.

19. Noone I, Fan CW, Tarrant H, O'Keefe S, McDonnell R, Crowe M: What happens to stroke patients after hospital discharge? Irish Medical Journal 200I, 94:15I-I52.

20. Department Of Health: Vascular Programme/Stroke. National Stroke Strategy. London, DH Publications; 2007.

21. Fan CW, McDonnell R, Johnson Z, O'Keeffe S, Crowe MJ: Hospitalbased stroke care in Ireland: results from one regional register. Irish Journal of Medical Science 2000, 169:30-33.

22. Hart JT: Community control of high blood pressure Edinburgh: Churchil Livingstone; 1987.

23. de Koning JS, Klazinga N, Koudstaal PJ, Prins AD, Borsboom G], Mackenbach JP: Quality of stroke prevention in general practice: relationship with practice organization. International Journal for Quality in Health Care 2005, 17(I):59-65.

24. Horgan F, Hickey A, Murphy S, Wiley M, Conroy R, McGee H, et al: First Irish National Audit of Stroke Care. Cerebrovascular Diseases 2007, 23(Suppl 2): 132

25. Strong K, Mathers C, Bonita R: Preventing stroke: saving lives around the world. Lancet Neurology 2007, 6:182-187.

26. Carandang R, Seshadri S, Beiser A, Kelly-Hayes M, Kase CS, Kannel WB, et al.: Trends in Incidence, Lifetime Risk, Severity, and 30-Day Mortality of Stroke Over the Past 50 Years. JAMA 2006, 296:2939-2946.

27. National Audit Office: Reducing Brain Damage: Faster access to better stroke care. London, The Stationary Office; 2005.

28. Hippisley-Cox J, Fenty J, Langford G, Pringle M, Coupland C: Quality of Care for Stroke and TIA in General Practice using the new GMS Contract Indicators. London, National Audit Office; 2005.
29. Heartwatch National Programme Centre \& Independent National Data Centre: Heartwatch Clinical Report. Dublin, Heartwatch National Steering Committee; 2006.

30. Miller C], Miller G, Fahridin S: Management of stroke in genera practice. Aust Fam Physician 2007, 36:890-89I.

31. Mayo NE, Nadeau L, Ahmed S, White C, Grad R, Huang A, et al.: Bridging the gap: the effectiveness of teaming a stroke coordinator with patient's personal physician on the outcome of stroke. Age And Ageing 2008, 37:32-38.

32. Zweifler J: The Missing Link: Improving Quality With a Chronic Disease Management Intervention for the Primary Care Office. Annals of Family Medicine 2007, 5:453-456.

\section{Pre-publication history}

The pre-publication history for this paper can be accessed here:

http://www.biomedcentral.com/1471-2296/10/27/pre pub

Publish with Biomed Central and every scientist can read your work free of charge

"BioMed Central will be the most significant development for disseminating the results of biomedical research in our lifetime. "

Sir Paul Nurse, Cancer Research UK

Your research papers will be:

- available free of charge to the entire biomedical community

- peer reviewed and published immediately upon acceptance

- cited in PubMed and archived on PubMed Central

- yours - you keep the copyright 